\title{
Importance for experiments in human medicine of imaging modalities for macroanatomical and histological study of rabbit suprarenal glands")
}

\author{
OMER GURKAN DILEK, ROSEN STEFANOV DIMITROV*, \\ KAMELIA DIMCHEVA STAMATOVA-YOVCHEVA*, \\ DAVID GOSPODINOV YOVCHEV*, RADOSLAV MIHAYLOV**
}

\author{
Department of Anatomy, Faculty of Veterinary Medicine, Mehmet Akif Ersoy University, Burdur, Turkey \\ *Department of Veterinary Anatomy, Histology and Embryology, Faculty of Veterinary Medicine, \\ Trakia University, Stara Zagora, Bulgaria \\ **Department of Animal Morphology, Physiology and Nutrition, Faculty of Agriculture, \\ Trakia University, 6000 Stara Zagora, Bulgaria
}

Dilek O. G., Dimitrov R. S., Stamatova-Yovcheva K. D., Yovchev D. G., Mihaylov R. Importance for experiments in human medicine of imaging modalities for macroanatomical and histological study of rabbit suprarenal glands

\section{Summary}

The morphological characteristics of the rabbit adrenal glands are currently investigated using routine imaging modalities. The aim of the study was to collect and interpret major findings and information in the literature on the rabbit as an animal model for investigations in humans. The suprarenal glands of thirty-four mature, clinically healthy New Zealand rabbits were studied using anatomical, routine histology, radiology, computed tomography, ultrasonography, and magnetic resonance imaging methods. The results demonstrated that the rabbit suprarenal glands are paired ellipsoid organs. The right gland was close to the right kidney, whereas the left gland was located at a distance from the left kidney. The capsule was composed of dense connective tissue. The parenchyma consisted of three zones: zona glomerulosa, zona fasciculate, and zona reticularis. The medulla was in the center of the glandular parenchyma. The glands' radiological and CT features defined their position relative to the right and left kidneys. The right suprarenal gland was with normal attenuation. The left suprarenal gland was located at a distance from the left kidney. The US features of the glands demonstrated variability in darkness and contrast, revealing specific histological features. The MRI peculiarities of the glands defined them as well visible findings.

Keywords: rabbit, suprarenal glands, imaging modalities, anatomy, histology

In modern human medicine, the rabbit is an appropriate animal model for scientific morphological and clinical investigations. New Zealand white rabbits are used for research experiments. They are calm and tolerate disorders better than other species. They are used as models for anatomical and histological studies, and for anatomical imaging research in humans (25). In addition, the anatomy of the New Zealand white rabbit's suprarenal glands is used to explore the alterations that occur in human suprarenal neoplasia (24).

The suprarenal glands in humans and mammals are paired organs located in the abdominal cavity. They

A part of this study was presented as a poster presentation at the $1^{\text {st }}$ International Health Science and Life Congress in Burdur, Turkey. May 2-5, 2017. are situated close to the kidneys. In some cases, the glands can be found cranial to both kidneys. Each gland is composed of a macroscopically visible capsule, a cortex, and a centrally located medulla. There are similarities in the location and topography of the glands in humans and rabbits. The rabbit adrenal glands are rose-gray ellipsoid elongated organs. Each suprarenal gland is located ahead of the cranial pole of the corresponding kidney. The glands are distinguished from the kidneys by the vessels that pass between them (4). A previous study (17) has shown the topography of the left and right suprarenal glands near the superior medial pole of the kidneys, whereas in humans, they are located above the superior poles. 
There are differences in the shape of the suprarenal glands in humans and other mammals. In humans, the glands are pyramidal in shape; the left is an incomplete pyramid, and the right is a typical pyramid. Each gland consists of a base, two surfaces (anterior and posterior), medial and lateral borders, and a pin. The base is narrow and ascends to reach the superior pole of the kidney, and at the medial border, the gland descends to reach the hilum of the kidney. The anterior surface of the right suprarenal gland is located close to the liver, on which it forms an impression. The posterior surface is orientated to the right crus of the diaphragm. Both crus of the diaphragm separate the glands at the $12^{\text {th }}$ thoracic vertebra on the right and the $1^{\text {st }}$ lumbar vertebra on the left. The medial border of the right suprarenal gland is close to the inferior vena cava. The left gland reaches the abdominal aorta (4).

Literature data concerning the anatomical and histological features of the rabbit suprarenal glands and their vascularisation are scarce. This is despite their morphological characteristics being widely applicable as a model for research on the human suprarenal glands $(13,32)$.

The macroscopic features of the suprarenal glands in domestic mammals show that both are composed of a cortex and a medulla. The glands are enveloped by a fine connective tissue capsule. The cortex and the medulla are macroscopically visible, since their color is different. Often the cortex is yellowish, and the medulla is reddish. The red color of the medulla is produced by the passage of the medullary vein (2).

A histological study of the goat suprarenal glands has been conducted in order to provide data on the morphological features of the glands in puberty and their development during sexual maturation. The study revealed that the goat suprarenal glands are enveloped by a two-layered capsule, and there are three well developed zones in the cortex: the zona glomerulosa, zona fasciculata, and zona reticularis. The widest zone is the zona fasciculata (10).

Two comparative anatomical and histological studies of the rabbit suprarenal glands have been performed to generate morphological data and to demonstrate the similarity of these glands to the human suprarenal glands $(15,28)$. Similarly to the human glands, the rabbit glands are paired retroperitoneal organs. They are embedded in fat tissue and are white or creamy in color. Their shape varies from oval to sickle. The glands are located superior to the superior pole of the kidney. A specific feature of the rabbit glands is that the left gland is located at a distance from the left kidney. The histological structure of these endocrine organs in mature animals reveals a two-layer capsule composed of a fibrous layer and a cellular layer. The parenchyma consists of three well-developed zones. The zona glomerulosa contains many sinusoids. In this zone, parenchyma cells are arranged in radiating cellular columns. These columns form arches with connective tissue of capsular origin in between. This connective tissue reaches the medulla. The zona fasciculata is the most developed zone, which extends largely in the cortex, between the zona glomerulosa and zona reticularis. It is composed of cubic cells distributed in cords. Between them, there are connective tissue strands with sinusoid capillaries. The zona reticularis is the third zone. Located between the medulla and zona fasciculata, it looks like a network because of the arrangement of its cells. The sinusoid capillaries are large and sinusoidal. There is no visible border between the medulla and zona reticularis. The centrally located medulla is constructed from chromaffin ovoid cells, which are grouped in cellular cords encircling the central vein.

Radiography has been used as an anatomical imaging method to study rabbit abdominal organs. Dorotea et al. (11) performed a radiological study and presented definitive anatomical data concerning the normal parameters of the rabbit kidneys and their topographic features and location in relation to the lumbar vertebrae.

Rabbit abdominal organs and large vessels have been studied anatomically by routine pre-contrast and post-contrast radiological methods. A post-contrast anatomical radiological study of rabbit abdominal and pelvic vessels has been conducted to obtain definitive and confirmative data about their topography (1).

Abdominal ultrasonography (US) has been used as an appropriate anatomical imaging modality to study the suprarenal glands in cats and ferrets and to generate anatomical data on the morphological features, shape, closeness, zonal structure, and topography of these glands in real time $(5,26,34)$.

With the introduction of the New Zealand white rabbit as an anatomical model for use in abdominal US investigations, some studies using US have already been published (3). One such study investigated the US peculiarities of the rabbit abdominal organs, especially both kidneys and the suprarenal glands, their morphometric parameters, and their relation to the stomach, as well as the small and large intestines. The US anatomical features of the rabbit abdominal vessels have also been investigated in real time (35). A two-dimensional US anatomical study of the rabbit kidneys has been performed to determine the morphological sonographic characteristics of these organs and their application in clinical practice (9). Furthermore, in recent years, abdominal US has been widely used to study the features of organs in living rodents and New Zealand rabbits, which are used as anatomical models. Anatomical structures have definitive topographic borders and are sharply distinguished from the adjacent soft tissues (20).

$\mathrm{CT}$ anatomical imaging has gained popularity as a definitive anatomical imaging modality to provide detailed knowledge of human abdominal organs and to diagnose relevant disorders using New Zealand 
white rabbits as an anatomical animal model. CT has been used to obtain retrospective anatomical images of the rabbit kidneys and to provide comprehensive anatomical information about these organs in surgical and morphological studies performed in humans (12).

CT soft-tissue findings are visualized as high-resolution structures. The reconstruction of certain images for a detailed study of parts of anatomical objects is a reason for computer tomography to be used as a live, non-invasive anatomical imaging method $(14,23)$.

Some researchers $(19,22,27)$ have investigated the capacity of some contrast agents for $\mathrm{CT}$ visualization of human abdominal organs. Therefore, they used the results of investigations in rabbits in their algorithms.

MRI is used as a live, non-invasive method that is appropriate for the contemporary interpretation of the anatomy of small pets and humans. An advantage of this method is its capability of creating three-dimensional reconstructions of organ structures, which provides a broad base of anatomical data, without any ionising effects (18).

The transference of magnetic relaxation times into gray and white signals of varying intensity has led to magnetic resonance tomography being used as the most appropriate method for the anatomical visualization of the heart, liver, digestive organs, and bones $(21,29)$.

The scarcity of data on the anatomical, histological, and anatomical imaging characteristics of the rabbit suprarenal glands was a motivation for the present study. The data obtained could be used as a morphological background for the contemporary interpretation of suprarenal gland anatomy in humans and rabbits, as well as for choosing appropriate anatomical imaging methods in humans, depending on experimental objectives.

\section{Material and methods}

Ethics statement. Permission for the use of animals in this study was given by the Ethical Committee for Animals of the Faculty of Veterinary Medicine at Trakia University in Stara Zagora (permissions No 51 of September 29, 2012, and No 59 of May 17, 2013). Experimental animals were purchased from the Agricultural Institute at Stara Zagora (ID No 1236503070041; VAT ID BG 123650307). Eighteen animals were delivered to the slaughterhouse Eurovarhat, which is licensed for the production and processing of lagomorph meat, in compliance with legal requirements concerning the rules at slaughter (Ordinance No 36 of March 23, 2006, promulgated in the State Gazette, issue 35 of April 28, 2006).

Materials. Thirty-four 8-month old (sexually mature) healthy white New Zealand rabbits weighing $2.8-3.2 \mathrm{~kg}$ were used. The animals were allocated to groups and methods, as shown in Table 1.

Tab. 1. Scheme of the experimental part: Methods and animals used

\begin{tabular}{|c|c|c|c|c|c|c|c|}
\hline Methods & NA & CAS & HIS & Radiol & 2D US & Helical CT & MRI \\
\hline Animals (rabbits) studied & $\mathbf{8}$ & $\mathbf{8}$ & $\mathbf{8}$ & $\mathbf{8}$ & $\mathbf{8}$ & $\mathbf{8}$ & $\mathbf{8}$ \\
\hline
\end{tabular}




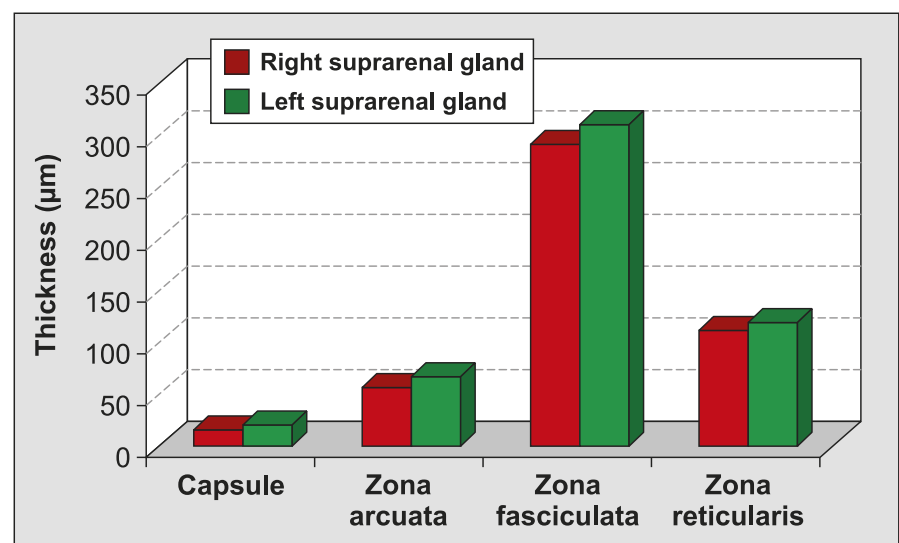

Fig. 1. Thickness of different zones in the rabbit suprarenal glands

$5 \mathrm{mAs}$ ). The spatial resolution was $10 \mathrm{px} / \mathrm{mm}$, the depth of scanning was $-20 \mathrm{bit} / \mathrm{px}$, and the depth after CPU processing was $16 \mathrm{bit} / \mathrm{px}$. We used software to read and measure the X-ray attenuated structure (iQ-VIEW Version 2.7.0 beta; IMAGE Information Systems Ltd, Rostock, Germany) (7).

US algorithm. The animals were in supine recumbency. For anesthesia, we used $15 \mathrm{mg} / \mathrm{kg}$ Zoletil $^{\circledR} 50$ (tiletamine $^{1}$ hydrochloride $125 \mathrm{mg}$ and zolazepam hydrochloride $125 \mathrm{mg}$ in $5 \mathrm{~mL}$ of solution) (Virbac, Carros-Cedex, France) intramuscularly. The investigation was performed using a Diagnostic Ultrasound System (model DC-6V; Mindray Bio-Medical, Electronics CO. LTD, Shenzhen, China). A microconvex probe $6 \mathrm{C} 2$ was used with a frequency of $7 \mathrm{MHz}$ and a radius of $20 \mathrm{~mm}$. For noninvasive monitoring, a contact gel (Ecoultragel; Pirrone \& Cö., Italy) was used. The US approach was transabdominal percutaneous.

CT algorithm. A coronal (dorsal) CT study was carried out. The animals were in supine recumbency. They were anaesthetized intramuscularly with $15 \mathrm{mg} / \mathrm{kg}$ Zoletil $^{\circledR} 50$ (tiletamine hydrochloride $125 \mathrm{mg}$ and zolazepam hydrochloride $125 \mathrm{mg}$ in $5 \mathrm{~mL}$ of solution) (Virbac, CarrosCedex, France). Helical CT scanning was performed with a high-resolution whole-body multi-slice helical computed tomography scanner (Light Speed QX/I GE; General Electric, Milwaukee, WI, USA). The body was scanned from the middle of the thorax to the pelvic inlet. The thickness of the CT slices was based on our previous study of the rabbit abdomen (30). The resolution was 512 pixels, the window width (WW) was 350 Hounsfield units (HU) and the window level (WL) was $35 \mathrm{HU}$. The slices of Regio abdominis were $5 \mathrm{~mm}$ thick. The study was performed on subsequent slices. The CT density of the right and left suprarenal glands was determined only for the purpose of adding detail to the protocol. The data were processed using DICOM and universal serial bus (USB) ports.

MRI algorithm. The rabbits were positioned in abdominal recumbency under deep anesthesia with Zoletil. Magnetic resonance tomography was performed using an MRI scanner (3 T Siemens Magnetom Essenza, Shenzen, China) with V-engine gradients $(30 \mathrm{mT} / \mathrm{m})$. The imaging was done in the coronal direction from the middle of the thorax to the pelvic inlet. The MRI system used reduced acquisition times with PAT factors until 8 . The magnetic weight was 3.5 tone and the strength of the magnetic field was $1.5 \mathrm{~T}$. The total imaging matrix (TIM) had 28 coil elements and 5 radiofrequency channels. The scanning diapason was up $140 \mathrm{~cm}$. The image reconstruction was 1131 images per second. The matrix was $256 / 256$. The thickness of the scans was 3 to $4 \mathrm{~mm}$. The field of view was $244 * 244$, time to echo (TE) was 93, and repetition time (TR) was 1410. The resolution was high.

\section{Results and discussion}

Native anatomical results. The rabbits' suprarenal glands were found to be retroperitoneal organs. They were enveloped by fat tissue which gave them a rose to creamy color. The adipose capsule of the right and left kidneys was separated from the fat capsule of both suprarenal glands. Their shape was ellipsoid (right gland) and oval (left gland). The right suprarenal gland was located craniomedially to the right kidney. The left one was situated craniomedially to the left kidney, but the distance between these two organs was larger than that between the right suprarenal gland and the right kidney. The caudal extremities of both glands were parallel to each other and situated in the same plane. An anatomical landmark for the position of and the distance between the glands and the kidneys were the right and left renal arteries, which passed across the median plane as the main branches of the abdominal aorta (Fig. 2).

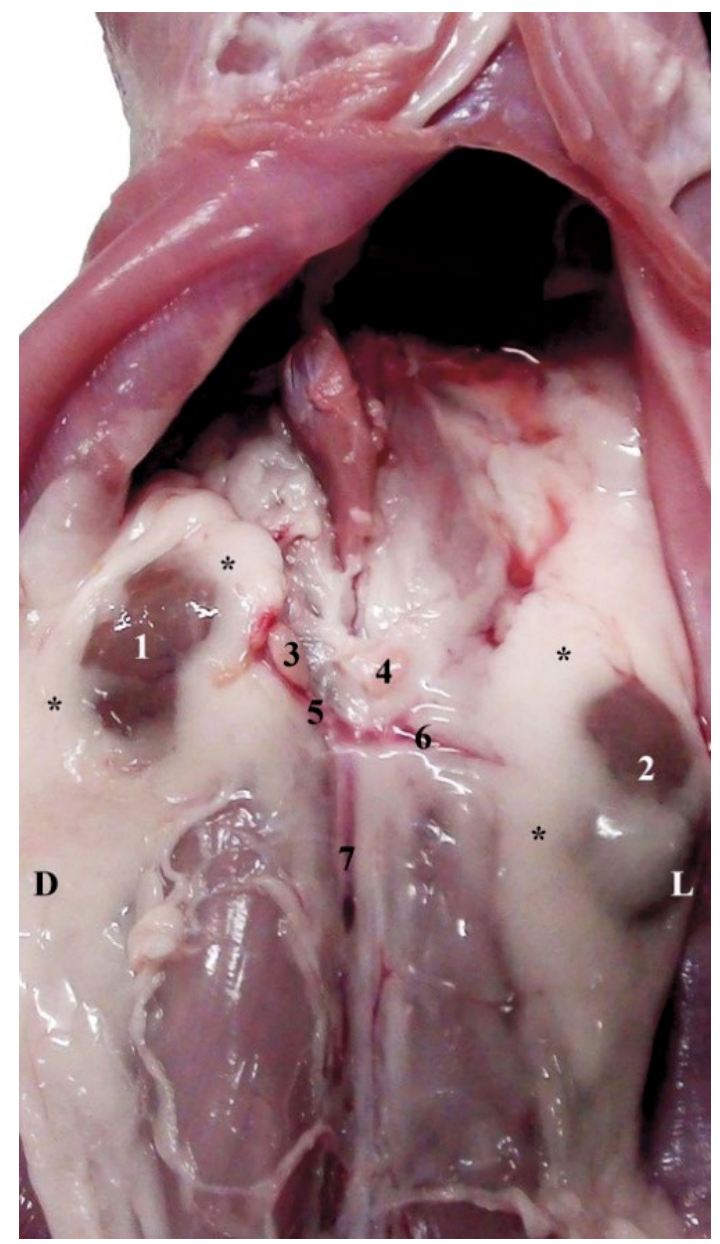

Fig. 2.

Explanations: D - right; L - left; (1) right kidney; (2) left kidney; (3) right suprarenal gland; (4) left suprarenal gland; (5) right renal artery; (6) right renal artery; (7) abdominal aorta; (*) adipose capsule 
Cross-sectional anatomical results. The transversal anatomical study of the rabbit abdominal cavity at the level of the first lumbar vertebra (L1) showed the closeness of the right suprarenal gland to the right kidney and the small intestine. The gland was found craniomedially to the right kidney. Ventrally, the gland had a close anatomical contact with the ascending part of the duodenum (pars ascendens duodeni) and ileum. In transverse cross-sectional cuts, the gland's shape was ellipsoidal with sharply distinguished borders toward the bean-shaped right kidney (Fig. 3).

Histological structure of the rabbit suprarenal glands. The histological study of the rabbit suprarenal glands provided detailed information about their microstructure. Both glands were composed of a capsule and parenchyma. The capsule was composed of dense connective tissue. The parenchyma consisted of three zones. The zona glomerulosa had sinusoid capillaries. In this zone, the cells were arranged in arch-like cellular strands with loose connective tissue in between. The zona fasciculata was the largest. It was found between the zona glomerulosa and the zona reticularis. It had cubic cells arranged in strings. Between them, there was loose connective tissue. The zona reticularis was the third zone. It resembled a network because of the irregular arrangement of its cells. The border between the zona reticularis and the medulla was not distinguished. The medulla was in the centre of the glandular parenchyma. It was composed of ovoid cells (Fig. 4).

The statistical analysis showed that the thickness of capsules of both glands was the smallest. The thickest component was the zona fasciculata, followed by the zona reticularis. The zona glomerulosa was the thinnest of the three parenchyma zones. The thickness of the zones was higher in the left suprarenal gland than it was in the right one (Fig. 1).

Radiological anatomical results. The native radiographic study of the suprarenal glands in the rabbit found that the opacity of both suprarenal glands was visible and distinguishable from the close soft tissue and bone structures. The radiological features of the glands defined their position relative to the right and left kidneys. The right suprarenal gland was visualized as soft tissue with intermediate attenuation, and its ventral portion was observed. The gland was oval and located close to the right kidney. The anatomical contact between the right suprarenal gland and the kidney was determined. The medial edge of the gland covered some of the right kidney. The left suprarenal gland was located at a distance from the left kidney. It had intermediate attenuation. It was oval (Fig. 5).

US anatomical results. The US study of the right half of the abdominal cavity in the lumbar region in the sagittal plane showed a normal US structure of the right suprarenal gland. In accordance with the greywhite scale, it was visualized as hyperechoic and oval, and could be sharply distinguished from the close soft tissue. Its contours were regular, hyperechoic, and

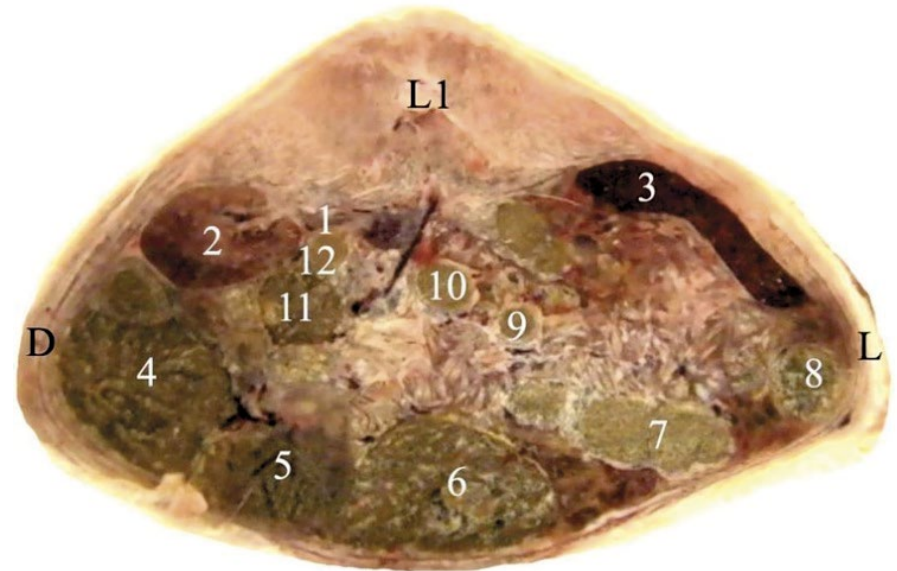

Fig. 3. Transverse anatomical cut at the level of $1^{\text {st }}$ lumbar vertebra (L1)

Explanations: (D) - right; L - left; (1) right suprarenal gland; (2) right kidney; (3) spleen; (4) haustral part of colon ascendens; (5) cecum; (6) non-haustral part of colon ascendens; (7) and (8) jejunum; (9) pars descendens duodeni; (10) pars transversa duodeni; (11) ileum; (12) pars ascendens duodeni

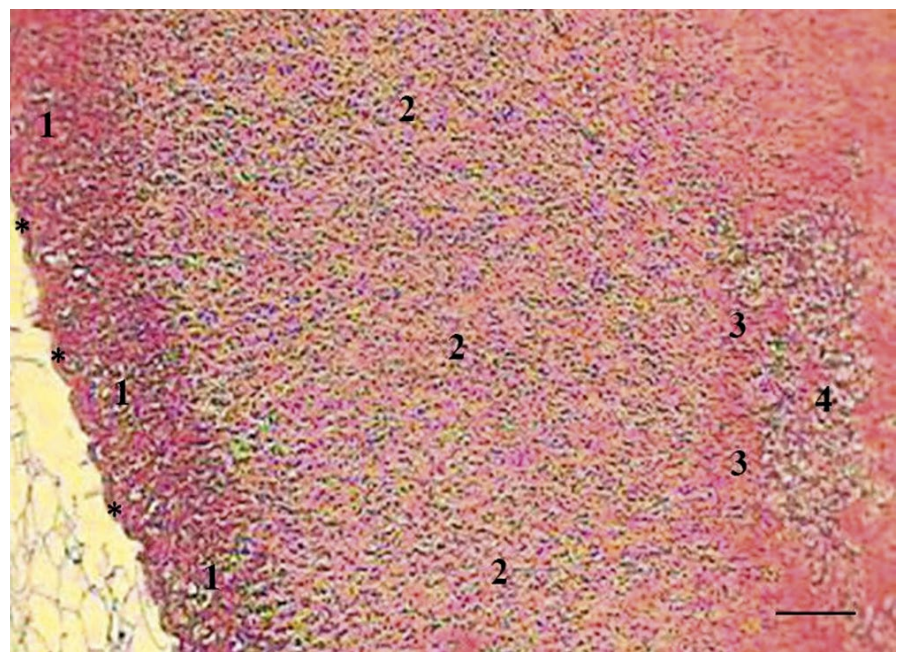

Fig. 4. Micro-image of the rabbit suprarenal glands (H/E) Explanations: $\left(^{*}\right.$ ) capsula fibrosa; (1) zona glomerulosa; (2) zona fasciculata; (3) zona reticularis; (4) medulla. Line $=50 \mu \mathrm{m}$

clearly visible. The gland was found craniomedially to the borders of the right kidney. The echogenicity of the gland was homogenous, with a fine grain character. The gland was observed at a short distance from the right kidney and was sharply defined by the relatively hypoechoic renal capsule, the adipose capsule of the kidney, and papilla renalis (Fig. 6).

Coronal (dorsal) CT anatomical results. The coronal CT anatomical image of the rabbit abdomen at a distance of $20 \mathrm{~mm}$ from the spine (analogic to native data) defined the CT visible distance between the left suprarenal gland and the corresponding kidney. Left of the median plane, the gland was visualized as an oval finding with intermediate attenuation. It was with an oval shape and sharply distinguished borders. It was located craniomedially to the cranial pole of the left kidney, and both structures were isodense. Some of the small intestinal segments were found close to the left suprarenal gland, since the border between them was 


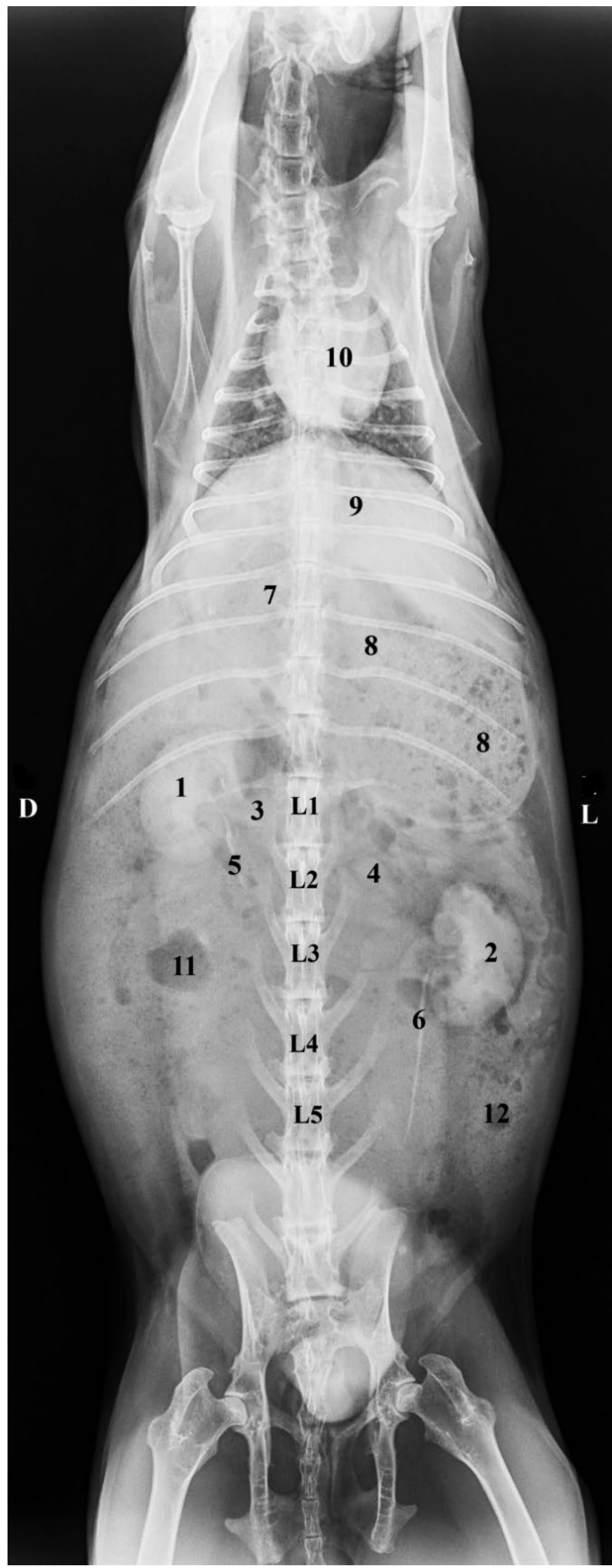

Fig. 5. Radiological image of the rabbit abdominal organs in supine recumbence

Explanations: D - right; L - left; (1) right kidney; (2) left kidney; (3) right suprarenal gland; (4) left suprarenal gland; (5) right ureter; (6) left ureter; (7) fundus ventriculi; (8) corpus ventriculi; (9) liver; (10) heart; (11) ascending colon; (12) descending colon determined by poorly visible hypoattenuated edges. This level of the scan did not define the anatomical location of the right suprarenal gland with respect to the right kidney (Fig. 7).

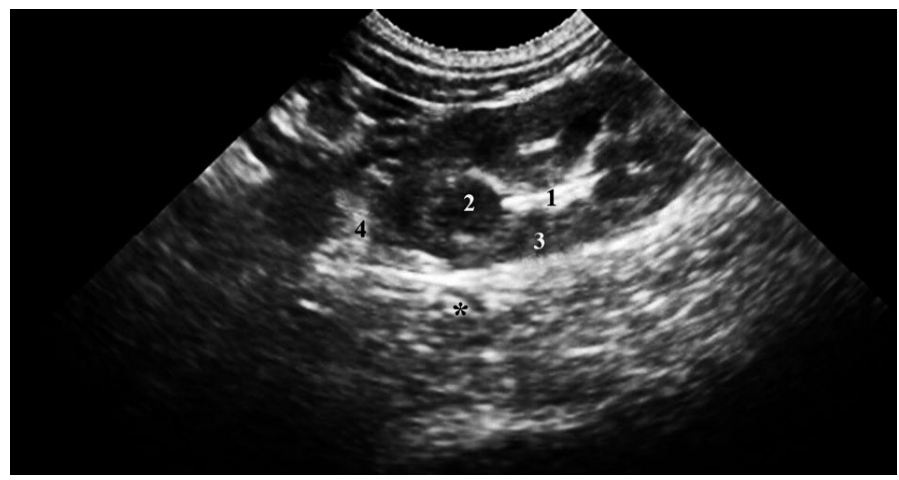

Fig. 6. Sagittal ultrasonographic anatomical image of the rabbit right adrenal gland (multifrequent microconvex transducer - 7 MHz; B-mode)

Explanations: (*) right adrenal gland; (2) papilla renalis; (3) cortex renalis; (4) capsula adiposa

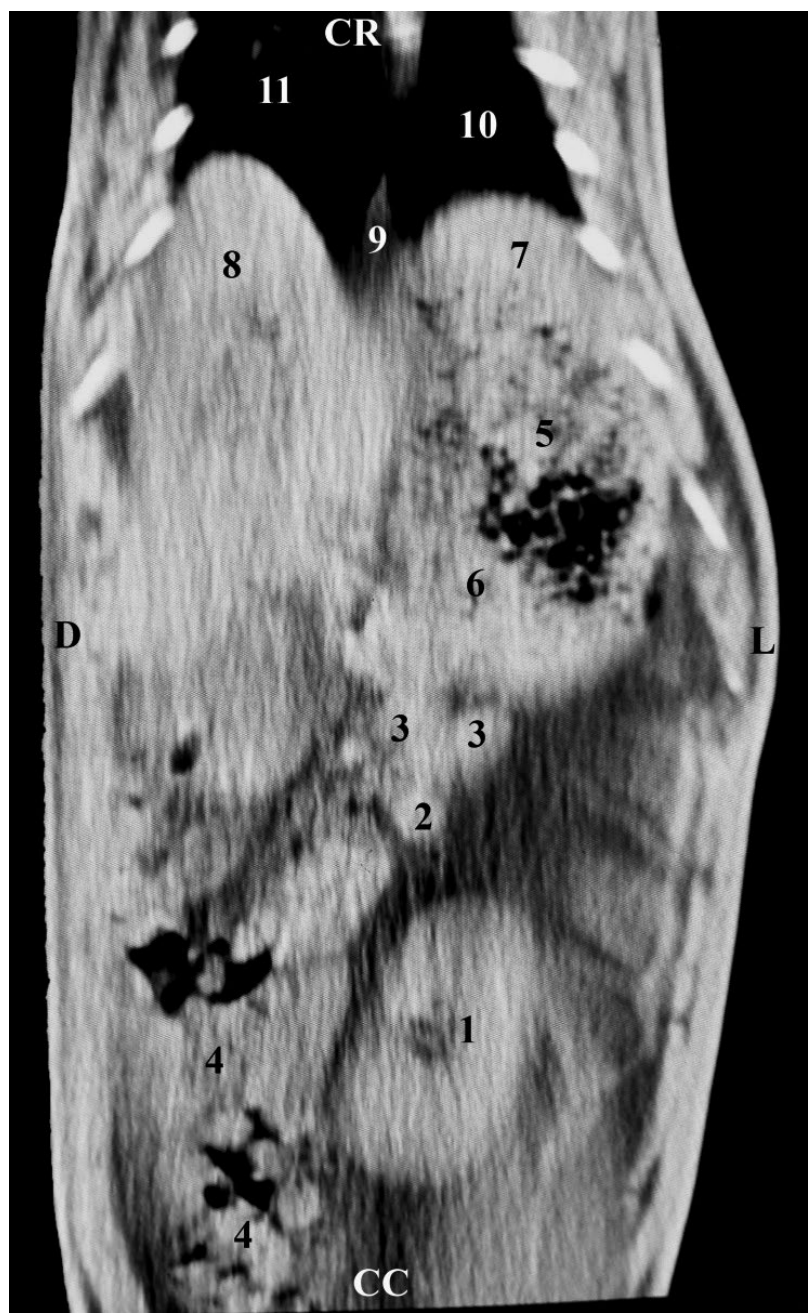

Fig. 7. Coronal (dorsal) CT anatomical image of the rabbit abdominal organs (The scan distance is $20 \mathrm{~mm}$ from the spine) Explanations: CR - cranial; CC - caudal; D - right; L - left; (1) left kidney; (2) left suprarenal gland; (3) small intestines; (4) ascending colon; (5) fundus of stomach; (6) body of stomach; (7) left lobe of liver; (8) right lobe of liver; (9) caudal vena cava; (10) left lung; (11) right lung 
MRI anatomical results. The dorsal MRI anatomical study of the rabbit abdominal organs at a distance of $20 \mathrm{~mm}$ from the spine and in a T1-weighted sequence defined the retroperitoneal position of both suprarenal glands. These were hyposignal findings. The contours of the glands had a lower signal and defined the ellipsoidal shape of the right gland and the oval shape of the left. The left and right glands were hyposignal compared to the right and left kidneys. The right suprarenal gland was located on the right of the median plane and craniomedially to the right kidney. The left one was situated on the left of the median plane and craniomedially to the left kidney. The distance between the left gland and kidney was larger than that between the right suprarenal gland and kidney (Fig. 8).

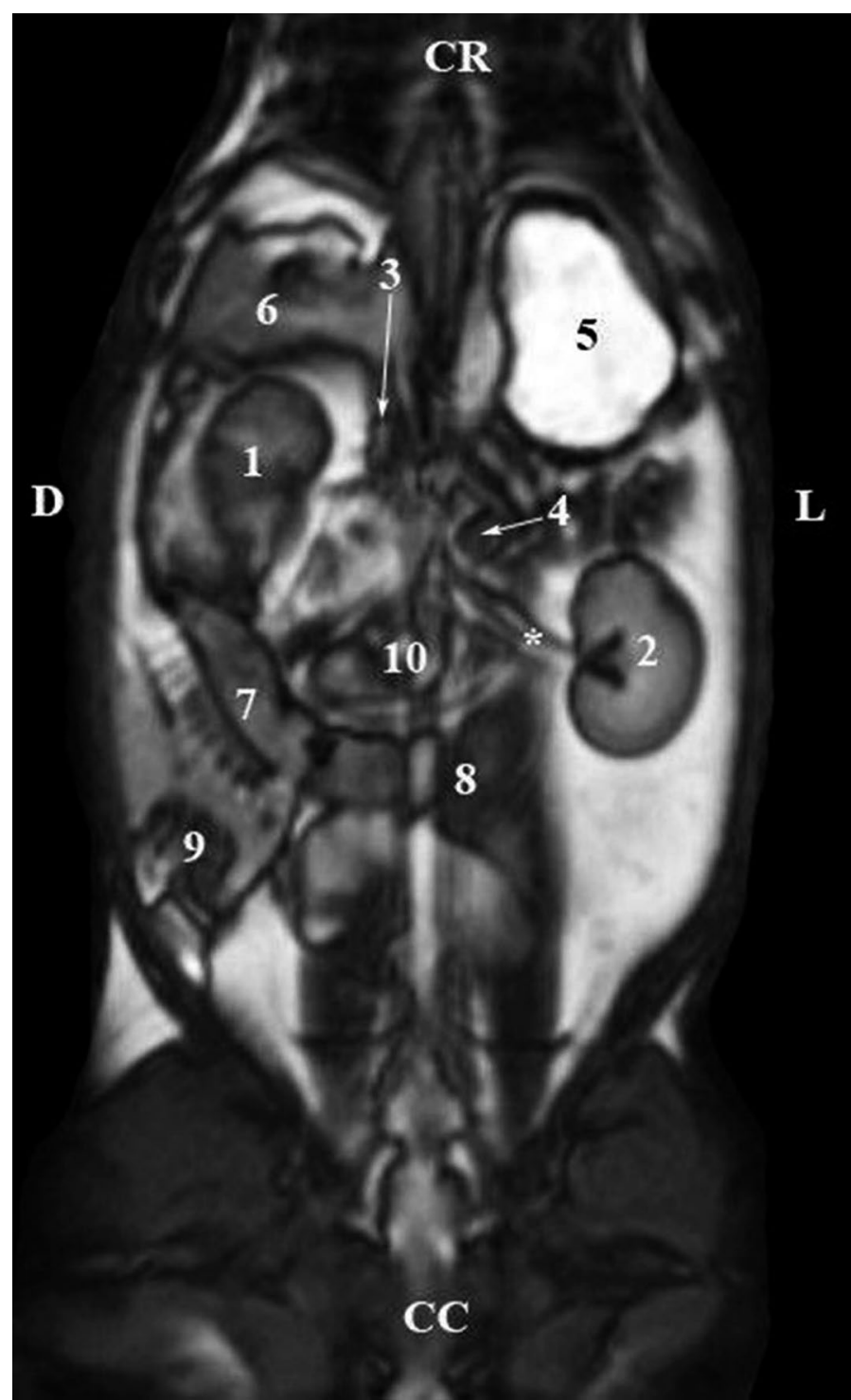

Fig. 8. Dorsal MRI anatomical image of the rabbit abdomina organs (T1-weighted sequence) (The scan distance is $30 \mathrm{~mm}$ from the spine)

Explanations: CR - cranial; CC - caudal; D - right; L - left; (1) left kidney; (2) left kidney; (3) right suprarenal gland; (4) left suprarenal gland; (5) body of stomach; (6) right lobe of liver; (7) ascending colon; (8) descending colon; (9) cecum; (10) small intestines
Our results from the anatomical study, as well as the anatomical imaging data for the rabbit suprarenal glands, describe them as paired organs with retroperitoneal topography in the abdominal cavity, and their proximities to the corresponding kidneys show a similarity in the location and topography of these glands in humans and rabbits. In addition, we obtained data on the macroscopic structure of these organs (capsule, cortex, and medulla), which is equivalent to that of the human suprarenal glands. Our interpretation corresponds to data published by some authors on the morphological characteristics of the rabbit suprarenal glands as well on their similarity to the human glands (4).

Our interpretation that both suprarenal glands in the rabbit are located craniomedially to the corresponding kidneys and that there is a larger visible distance between the left gland and kidney than there is between the right gland and kidney differs from the published data (17) on these organs in animals. In addition, we used the right and left renal arteries as anatomical landmarks for the position and the relationship between the glands and kidneys.

Despite the many similarities between the rabbit and human suprarenal glands, we also identified some differences. Our results concerning the glands' shape (oval for the left gland and ellipsoid for the right one) demonstrated that there are differences between the shape of the rabbit suprarenal glands and the shape of the human glands (typical pyramidal organs) (4). In addition, we identified the anatomical relationship of the suprarenal glands to the kidneys and described the closeness of the right gland to the right kidney and the distance between the left gland and the left kidney. We did not find anatomical contact between the right gland and the liver or diaphragm, which differed from the published data on the human suprarenal glands (4). Furthermore, we suggested additional anatomical landmarks to describe the topography of the glands: both renal arteries. We did not describe the proximity of the right gland to the caudal vena cava or that of the left gland to the abdominal aorta, which contradicts the literature data on the human glands (4). We excluded the diaphragm as a possible landmark, as described for the human suprarenal glands. The data demonstrate anatomical differences between the glands in rabbits and humans (4).

The use of the rabbit suprarenal glands as a model in morphological research on the human suprarenal glands prompted us to conduct our study using different approaches, from anatomical and histological to anatomical imaging (2D US, CT, Radiology, and MRI). Our data will add to the knowledge and provide an innovative and modern interpretation of the morphology of the rabbit suprarenal glands $(13,32)$. We demonstrated the presence of capsule and three welldeveloped zones in the cortex: zona glomerulosa, zona 
fasciculata, and zona reticularis, which corresponds to the published data (10) on the same glands in the goat. Additionally, we found that the thickest zone was the zona fasciculata.

Our algorithm of investigation included anatomical, histological, and anatomical imaging studies. Like the suprarenal glands in humans, these organs in rabbits are retroperitoneal and embedded in fat tissue; they are proximal to the corresponding kidneys, although the left is visibly distanced. There are histological resemblances with the human glands, since we also found a well-developed capsule and parenchyma in the rabbit glands. There are three parenchyma zones in the rabbit glands, as there are in the human glands, and the arrangement of these zones is similar: the zona glomeruloza is peripheral with sinusoid capillaries, the zona fasciculata is the middle one, and the reticular zone resembles a network, close to the medulla. Similarly to the human suprarenal glands, we did not find a border between the third parenchyma zone and the medulla. These findings suggest that the rabbit suprarenal glands are a suitable anatomical and histological model to study the same glands in humans. This is in line with the views of some authors, e.g. Rabie (28) and Hussein and Abass (15), who have investigated the rabbit suprarenal glands.

Our radiological algorithm focused on the description of the topography of the rabbit suprarenal glands. We described their adjacency to the close soft tissue (both kidneys) and obtained definitive anatomical data on the features of the gland, according to the level of $\mathrm{X}$-ray attenuation. We propose that radiology is an appropriate anatomical imaging method for studying the rabbit suprarenal glands. This adds to the published data on the application of this method in the anatomical study of rabbit abdominal organs (11).

Our radiological algorithm focused on the precontrast study of the rabbit supernal glands. Therefore, we consider the rabbit a suitable animal model for radiological investigations. However, we investigated only the suprarenal glands (parenchyma organs), and did not perform a post-contrast study. In this respect, our algorithm differed from the studies of rabbit vessels using the post-contrast approach (1).

We also suggest that abdominal ultrasonography (US) is an appropriate anatomical imaging modality for studying the suprarenal glands in the rabbit. We noted the advantages of real-time US in studying the closeness of the organs as well as their shape and topography, according to variations in the grey-white scale. This is in line with data on the use of US as an anatomical imaging method in other small mammals $(5,26,34)$.

We believe that a 2D US study is a suitable live and non-invasive method for studing the topography of the rabbit suprarenal glands. We identified the US characteristics of the glands (their shape, acousticity, echoic character, closeness, borders, and sharpness), which corroborated previous studies of rabbit abdominal organs $(3,4,9,35)$.

Our pre-contrast CT helical images of the rabbit suprarenal glands had a high resolution. This is a prerequisite for a detailed anatomical visualization using a retrospective reconstruction of these organs. The data could be used as a model for the interpretation of live anatomical imaging of human suprarenal glands. The above-mentioned facts motivated us to corroborate the results of some authors (12) for the rabbit.

The helical CT results for the rabbit suprarenal glands add to the exiting literature data $(14,23)$ on the application of this method to obtain naturalistic, high-resolution anatomical images. In addition, this method can be used on live animals.

Our hypothesis motivated us to suggest that our results could be used as an anatomical imaging model for studying the CT anatomical features of the human suprarenal glands. These observations are in agreement with data of some authors $(19,22,27)$ for the rabbit.

The transference of magnetic relaxation times into gray and white signals of varying intensity causes magnetic resonance tomography to be used as the most appropriate method for the anatomical visualization of the heart, liver, digestive organs, and bones $(21,29)$.

The MRI anatomical images of the rabbit suprarenal glands were reconstructed automatically during the study. We believe that this type of tomography is a suitable anatomical method for the visualization of these organs (18).

MRI anatomical visualization of the rabbit suprarenal glands in a T1-weighted sequence was made possible by variations in the magnetic signal and the tissue contrast of the glands and the close soft tissue findings (both kidneys). The anatomical objects were reconstructed in the dorsal (coronal) plane. Our conclusion is that MRI is a highly informative anatomical imaging method because the images have a high resolution. Therefore, we agree with some other authors (21, 29) about the usefulness of this method.

The results of the MRI study led us to conclude that the rabbit suprarenal glands are a suitable experimental model for MRI studies. Thus, we confirm literature data (16) according to which the rabbit is an appropriate experimental model for MRI studies in human medicine.

The results of our morphological and anatomical imaging studies provided a detailed description of the retroperitoneal anatomical location of the rabbit suprarenal glands in relation to the right and left kidneys. In addition, we described the histological characteristics of the glands. This analysis could be used as a basic anatomical alternative to study the human suprarenal glands $(24,25)$.

The comparative analysis of data from post mortem and anatomical imaging studies led us to conclude that the imaging methods used (radiology; 2D US, 
helical CT, and MRI) are adequate and satisfactory methods for the anatomical interpretation of the rabbit suprarenal glands in living subjects. In addition, anatomical data from either post mortem studies or anatomical imaging investigations could be used as a morphological basis for investigations of the human suprarenal glands.

\section{References}

1.Abidu-Figueiredo M., Xavier-Silva B., Cardinot T., Babinski M., Chagas A. Celiac artery in New Zealand rabbit: anatomical study of its origin and arrangement for experimental research and surgical practice. Pesqui. Vet. Bras. 2008, 28, 237-240.

2. Ahmadpanahi J.: Anatomical and Histological Studies of Accessory Adrenal Nodules in Caspian Miniature Horses. Turk. J. Vet. Anim. Sci. 2007, 31, $275-$ -278 .

3. Banzato T., Bellini L., Contiero B., Selleri P., Zotti A.: Abdominal ultrasound features and reference values in 21 healthy rabbits. Vet. Rec. 2015, 176, 101. doi: 10.1136/vr.102657.

4. Barone R., Simoens P.: Glandes surrénales, [in:] Anatomie Comparée des Mammifères Domestiques, tome 7: neurologie, II: système nerveux périphérique; glandes endocrines; esthésiologie. Edition Vigot, Paris 2010.

5. Besso J. G., Tidwell A. S., Gliatto J. M.: Retrospective review of the ultrasonographic features of adrenal lesions in 21 ferrets. Vet. Radiol. Ultrasound 2005, 41, 345-352

6. Dimitrov R.: Morphofunctional and imaging features of the male accessories glands and pelvic part of the urethra in the tomcat. $\mathrm{PhD}$ Thesis, Trakia University, Stara Zagora, Bulgaria 2009.

7. Dimitrov R., Chaprazov T.: An anatomic and contrast enhanced radiographic investigation of the rabbit kidneys, ureters and urinary bladder. Rev. Med. Vet. 2012, 163, 469-474.

8. Dimitrov R., Kostov D., Stamatova K., Yordanova V.: Anatomotopographical and morphological analysis of normal kidneys of rabbit (Oryctolagus cuniculus). Trakia J. Sci. 2012, 10, 79-84.

9. Dimitrov R. S.: Ultrasound features of kidneys in the rabbit (Oryctolagus cuniculus). Vet. World 2012, 5, 274-278.

10. Dimova T.: Histological features of goat adrenal gland during puberty. C. R. Acad. Bulg. Sci. 2003, 56, 91-96

11. Dorotea S. B., Banzato T., Bellini L., Contiero B., Zotti A.: Radiographic anatomy of dwarf rabbit abdomen with normal measurements. BJVM 2016, 19, 96-107.

12.Eken E., Çorumluoglu Ö., Paksoy Y., Beşoluk K., Kalaycı I.: A study on evaluation of $3 \mathrm{D}$ virtual rabbit kidney models by multidetector computed tomography images. Anatomy 2009, 3, 40-44, doi:10.2399/ana.09.009.

13. Harrison R. G.: A comparative study of the vascularization of the adrenal gland in the rabbit, rat and cat. J. Anat. 1951, 85, 12-23.

14. Henninger W., Frame M., William M.: CT features of alveolotis and sinusitis in horses. Vet. Radiol. Ultrasound 2003, 44, 269-276.

15. Hussein A. A., Abass T. A.: Comparative anatomical and histological study of the rabbit (Oryctolagus cuniculus). DASJ 2015, 7, (2), 1-11.

16. Hynynen K., Damianou C., Colucci V., Unger E.: MR monitoring of focused ultrasonic surgery of renal cortex: experimental and simulation studies. J. Magn. Reson. Imaging 2005, 5, 259-266.

17. Karakurum E., Özgel Ö., Dursun N.: Morphology and Arterial Vasculature of Donkey (Equus asinus L.) Adrenal Gland. Turk. J. Vet. Anim. Sci. 2008, 32, 469-473.

18. Kastler B., Vetter D., Patay Z., Germain P. eds.: Comprendre 1'IRM: manuel d'auto-apprentissage, $4^{\text {th }}$ ed. Paris: Masson Bulgarian translation by Prof. Vassil Hadjidekov, Physical Principles of MRI. Medi. Phys. Education Sofia 2005 , p. 23.

19. Krause W., Schönborn A., Rupp K.: CT imaging with iopromide liposomes in a rabbit model. J. Liposome Res. 2011, 21, 229-236.

20.Kressl M., Becker K., Schloegl S., Schlotter H., Kaiser F., Reiners C., Lassmann M.: Multimodal small animal imaging: Combination of 3D-ultrasound and small animal Pet/Spect. J. Nucl. Med. 2009, 50, 295.

21. Lauridsen H., Hansen K., Wang T., Agger P., Andersen J., Knudsen P., Rasmussen A., Uhrenholt L., Pedersen M.: Inside out: Modern Imaging Techniques to Reveal Animal Anatomy. Plos One 2011, 6, (3), e17879, doi: 10.1371/journal.pone.0017879.

22. Leander P., Mansson S., Ege T., Besjakov J.: CT and MR imaging of the liver using liver-specific contrast media. A comparative study in a tumour model. Acta Radiol. 1996, 37, 242-249.
23. Lenard Z.: New Advances In Small Animal Imaging - CT. Small Animal Medicine Chapter, [in:] ACVSc College Science Week. Murdoch University 2008, p. $70-73$

24. Lennox M. A., Chitty J.: Adrenal neoplasia and hyperplasia as a cause of hypertestosteronism in two rabbits. Journal Exot. Pet Med. 2006, 15, 56-58.

25. Mapara M., Thomas B. S., Bhat K. M.: Rabbit as an animal model for experimental research. Dent. Res. J. 2012, 9, 111-118.

26. O'Brien R. T., Paul-Murphy J., Dubielzig R. R.: Ultrasonography of adrenal glands in normal ferrets. Vet. Radiol. Ultrasound 1996, 37, 445-448.

27. Okada M., Isoda T., Kumano S., Kagawa Y., Araki T., Onishi H., Hori M. Kim T., Motokui Y., Wada T., Murakami T.: Serine- and mannose-modified liposomal contrast agent for computed tomography: evaluation of the enhancement in rabbit liver VX-2 tumor model. Contrast Media Mol. I. 2010, 5, 140-146.

28. Rabie F. O.: Morphological study of adrenal gland in prenatal and postnatal periods of domestic rabbit (Oryctolagus cuniculus). Bas. J. Vet. Res. 2013, $12,253-264$

29. Soler M., Murciano J., Latorre R., Belda E., Rodriguez M., Agut A.: Ultrasonographic, computed tomographic and magnetic resonance imaging anatomy of the normal canine stifle joint. Vet. J. 2007, 174, 351-361.

30. Stamatova-Yovcheva K.: The application of some imaging anatomical methods for investigation of the liver of the New Zealand white rabbit (Oryctolagus cuniculus). PhD thesis. Stara Zagora, Bulgaria 2016.

31. StatSoft ${ }^{\mathbb{B}}$ Statistica 7: Descriptive statistic. Dell Software, USA 2013.

32. Toru K., Brown M. S., Watanabe Y., Goldstein J. L.: Deficiency of low density lipoprotein receptors in liver and adrenal gland of the WHHL rabbit, an animal model of familial hypercholesterolemia. Proc. Natl. Acad. Sci. 1981, $78,2268-2272$

33. Vitanov S., Dimitrov D., Bochukov A.: Methods for investigation in Cytology and Histology, [in:] Popova M. (ed.). Zemizdat EOOD, Sofia 1995, p. 3-22.

34. Zatelli A., D'Ippolito P., Fiore I., Zini E.: Ultrasonographic evaluation of the size of the adrenal glands of 24 diseased cats without endocrinopathies. Vet. Rec. 2007, 160, 658-660.

35. Zhang L., Duan Y., Yin J., Cui J., Zhang Y., Cao T.: Gray scale enhancement by a new self-made contrast agent in early cirrhotic stage of rabbit liver. BMC Gastroenterol. 2007, 7, 32

Corresponding author: Omer Gurkan Dilek, DVM, Assist. Prof. Dr., Department of Anatomy, Faculty of Veterinary Medicine, Mehmet Akif Ersoy University, Burdur, Turkey; e-mail: ogdilek@gmail.com 